\title{
Episodio de tiempo severo con elevado impacto en Cataluña del 18 de octubre de 2017
}

https://doi.org/10.31978/639-19-010-0.473

\author{
Ramón Pascual Berghaenel ${ }^{1}$ (rpascualb@aemet.es) \\ Gabriela Cuevas Tascón ${ }^{1}$ (gcuevast@aemet.es)
}

${ }^{1}$ AEMET / Delegación Territorial en Cataluña

\begin{abstract}
RESUMEN
El 18 de octubre de 2017 se produjo en Cataluña un episodio de tiempo severo, que afectó principalmente a la provincia de Tarragona, con lluvias torrenciales e inundaciones repentinas, gran aparato eléctrico, rachas de viento muy fuertes e incluso huracanadas y, por lo menos, un probable tornado en la localidad de Valls (Alt Camp). Estos fenómenos tuvieron un elevado impacto con daños materiales de diferente consideración y algunos heridos.

La situación meteorológica a escala sinóptica en el entorno ibérico estaba caracterizada por una profunda vaguada de eje norte-sur, especialmente clara en 500 y $300 \mathrm{hPa}$, y un par de frentes fríos cruzando de oeste a este la Península. La vaguada iba asociada a una masa fría con valores térmicos mínimos de $-22{ }^{\circ} \mathrm{C}$ a $500 \mathrm{hPa}$. En este entorno se desarrolló en el nordeste peninsular convección organizada en forma de línea de turbonada en el extremo sur de la cual se formó a su vez un bow echo, responsable de los vientos más violentos. Este estudio analiza la fenomenología y los efectos asociados, el entorno sinóptico y mesoescalar y la convección generada, así como los avisos de fenómenos meteorológicos adversos emitidos.
\end{abstract}

PALABRAS CLAVE: convección profunda; convección organizada; severidad; riesgo meteorológico; impacto.

\section{INTRODUCCIÓN}

Este estudio analiza el episodio de lluvias torrenciales y rachas fuertes de viento del día 18 de octubre de 2017, que tuvo un fuerte impacto especialmente en el nordeste peninsular. Dicho episodio tuvo lugar dentro de una situación de precipitaciones generalizadas en la península ibérica, especialmente en el suroeste y en el nordeste peninsular, acompañadas de un elevadísimo número de descargas eléctricas.

El entorno sinóptico vino determinado por el paso de una profunda vaguada en altura (en 300 y $500 \mathrm{hPa}$ ) con un mínimo de $-22^{\circ} \mathrm{C}$ en $500 \mathrm{hPa}$, y con dos frentes fríos muy activos asociados que fueron atravesando la Península de oeste a este a lo largo de ese día. Estos frentes actuaron como mecanismo de disparo de la convección primaria. En el nordeste peninsular la convección fue relevante, con la formación de una larga estructura lineal organizada (13 UTC, al sur de Cataluña) que dio origen a una línea de turbonada (con un bow echo en su parte sur). Esta línea tuvo un importante impacto en la provincia de Tarragona, donde causó graves daños materiales, múltiples heridos, decenas de árboles caídos, cortes de suministro eléctrico, carreteras y trenes... En el municipio de Valls, donde se produjeron los mayores daños, es muy probable que se formara un tornado (BECH et al., 2018). 
Los episodios altamente convectivos en la península ibérica se dan especialmente en las áreas cercanas al Mediterráneo durante los meses de verano y otoño donde la orografía provoca que el aire húmedo mediterráneo ascienda rápidamente (Romero et al., 1998; Doswell III et al., 1998; RoMERO et al., 1999; RIESCO et al., 2014). Cuando las condiciones atmosféricas favorecen un cierto nivel de organización de la convección y se generan sistemas convectivos de mesoescala (MCS) (RIOSALIDO et al., 1998; HouZE, 2004; MATEO et al., 2009) o sistemas multicelulares o supercélulas (DAVIES-JONES, 2015), la probabilidad de que se produzcan distintos tipos de fenómenos severos aumenta notablemente (PASCuAL et al., 2015). Las líneas de turbonada son la forma más frecuentemente observada de MCS y no son inusuales en España, siendo habitual que tengan cientos de kilómetros de longitud (ÁLVAREZ et al., 2018).

Los sistemas convectivos de mesoescala pueden reforzar la posibilidad de vientos severos en superficie (KuCHERA et al., 2005). Estos eventos también pueden producir inundaciones repentinas, que conllevan un alto impacto en la sociedad, especialmente cuando ocurren en zonas pobladas (LlASAT et al., 2010). En Cataluña, el otoño es la estación que concentra la mayor ocurrencia de inundaciones.

\section{OBSERVACIONES}

El episodio convectivo analizado dio lugar a chubascos de intensidad fuerte o muy fuerte y localmente torrencial y a rachas de viento muy fuertes y probablemente huracanadas en distintos puntos. También es muy probable que se registrara un tornado EF1 en la localidad de Valls (BECH et al., 2018).

Aunque los chubascos fuertes afectaron a diferentes comarcas de la provincia de Tarragona, las mayores intensidades y acumulaciones se registraron en las comarcas de la Terra Alta y el Priorat, ambas ocupando parte del ámbito geográfico de la cordillera prelitoral.

Se listan a continuación las precipitaciones más destacables (> $40 \mathrm{~mm}$ ) (horas UTC) asociadas al episodio analizado (aunque en otros momentos del día también se registraron chubascos):

\section{Comarca: Terra Alta}

Gandesa: $113 \mathrm{~mm}(10: 30-13: 30) .59 \mathrm{~mm} / 60 \mathrm{~min}(12: 30-13: 30)$ (muy fuerte-torrencial).

Horta de Sant Joan: $70 \mathrm{~mm} .61 \mathrm{~mm} / 60 \mathrm{~min}$ (10:20-11:20) (torrencial) (está asociada a una célula convectiva anterior al episodio analizado).

Parc Natural dels Ports: 57 mm. 29 mm/60 min (10:30-11:30) (fuerte) (está asociada a una célula convectiva anterior al episodio analizado).

\section{Comarca: Priorat}

Margalef: $63 \mathrm{~mm} .44 \mathrm{~mm} / 60 \mathrm{~min}(14: 30-15: 30)$ (muy fuerte).

Ulldemolins: $73 \mathrm{~mm} .43 \mathrm{~mm} / 60 \mathrm{~min}(14: 50-15: 50)$ (muy fuerte).

Pantà de Siurana: $70 \mathrm{~mm} .47 \mathrm{~mm} / 60 \mathrm{~min}$ (14:50-15:50) (muy fuerte).

Falset: $61 \mathrm{~mm} .28 \mathrm{~mm} / 60 \mathrm{~min}(14: 30-15: 30)$ (fuerte).

Torroja del Priorat: $55 \mathrm{~mm} .21 \mathrm{~mm} / 60 \mathrm{~min}$ (14:50-15:50) (fuerte).

Cabacés: $41 \mathrm{~mm} .31 \mathrm{~mm} / 60 \mathrm{~min}(14: 30-15: 30)$ (muy fuerte).

En puntos de la zona afectada por los vientos fuertes (comarcas del Alt Camp, Baix Camp, Tarragonès y Baix Penedès) se registraron también intensidades torrenciales (calculadas en periodos de $10 \mathrm{~min}$ ) pero acumulaciones totales relativamente pequeñas.

Se listan ahora por comarcas las mayores rachas de viento registradas por distintas redes en la provincia de Tarragona. En el caso de conocerse, se indica la hora (UTC) de la racha máxima. 
Comarca: Priorat

Siurana: $75 \mathrm{~km} / \mathrm{h}$ a las 15:51.

Comarca: Baix Camp

La Mussara: $81 \mathrm{~km} / \mathrm{h}$.

L'Albiol: $72 \mathrm{~km} / \mathrm{h}$.

Almoster: $74 \mathrm{~km} / \mathrm{h}$.

Aeropuerto de Reus: 71 km/h a las 16:00.

Comarca: Tarragonés

Constantí: $113 \mathrm{~km} / \mathrm{h}$.

Tarragona (1): $73 \mathrm{~km} / \mathrm{h}$ a las 15:47.

Tarragona (2): $101 \mathrm{~km} / \mathrm{h}$ a las 16:00.

Torredembarra: $114 \mathrm{~km} / \mathrm{h}$ a las 15:58.

Comarca: Alt Camp

Vallmoll: $81 \mathrm{~km} / \mathrm{h}$ a las 16:05.

Valls: $118 \mathrm{~km} / \mathrm{h}$ a las 16:07 (esta racha podría estar asociada al probable tornado).

Vila-rodona: $99 \mathrm{~km} / \mathrm{h}$ a las 16:07.

Comarca: Baix Penedés

El Montmell: $81 \mathrm{~km} / \mathrm{h}$ a las 16:18.

EI Vendrell: $77 \mathrm{~km} / \mathrm{h}$.

Fuentes de datos de precipitación y viento: AEMET, SMC y Meteoclimatic.

Aunque hubo distintas estructuras mesoescalares que dieron lugar a los fuertes vientos, es posible seguir, a partir de los datos de las estaciones meteorológicas automáticas (EMA), el avance de sudoeste a nordeste del frente de racha asociado al sistema convectivo. La fig. 1 muestra la evolución temporal de la temperatura del aire, el punto de rocío, la velocidad media del viento y el valor de la racha máxima (calculadas en periodos de 10 min) para tres EMA de la provincia de Tarragona y una de Barcelona, situada algo más al norte que las anteriores. En todas ellas se observa una caída de la temperatura y del punto de rocío hacia las 16:00 UTC coincidiendo con una fuerte y aislada racha de viento. Tras este brusco cambio la temperatura se mantuvo notablemente más baja, la humedad relativa más alta y la velocidad del viento menor. La brusquedad del cambio térmico y las elevadas y momentáneas rachas de viento indican que, aunque los fenómenos convectivos se sucedieron al paso de un frente frío, no fue este sino un potente frente de racha mesoescalar el que dio lugar dichos cambios.

Los datos de descargas eléctricas nube-tierra (NT) procedentes de la red de detección de AEMET muestran, a escala subsinóptica, el avance oeste-este de las estructuras convectivas generadas a lo largo del doble frente frío (fig. 5b) que fue cruzando la península ibérica. A modo de ejemplo, entre las 12 y las 14 UTC (fig. 2) las tormentas se extendían entre el Pirineo de Huesca y la costa de Murcia, a lo largo de casi $600 \mathrm{~km}$. Este hecho muestra el favorable entorno convectivo presente en la mitad oriental peninsular y la eficacia de este frente frío como disparador de la convección. 

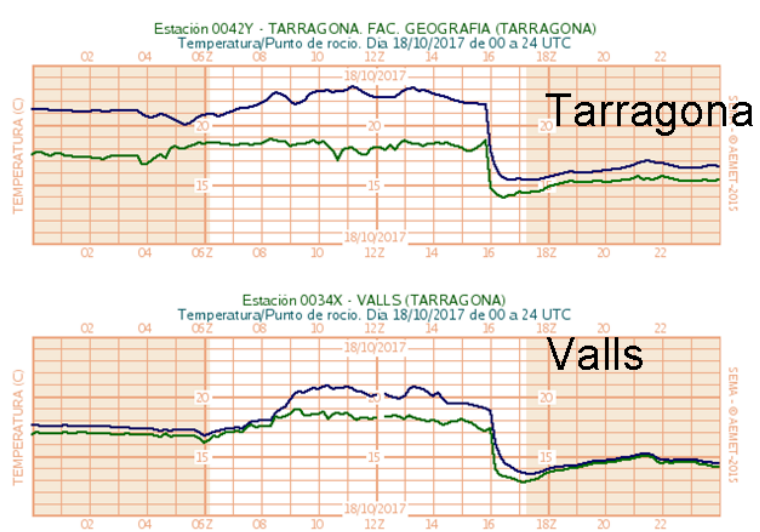

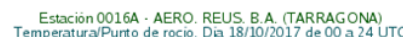
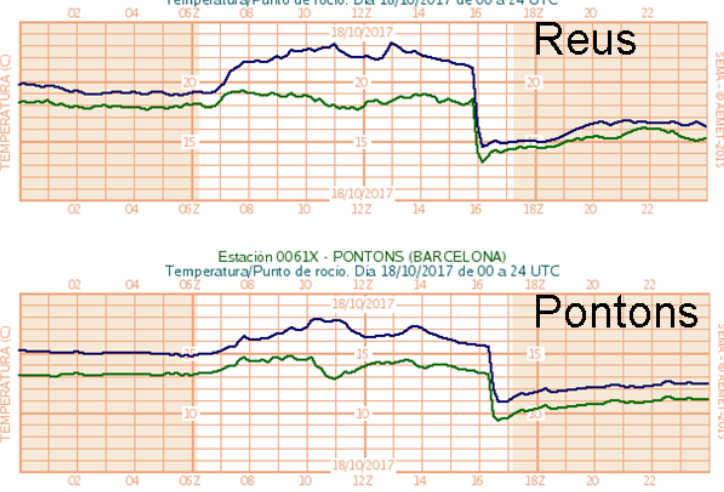

Estacion OOA2Y. TARRAGONA FAC. GEOGRAFAA (TARRAGONA)
Vel. media del viento Y Maximo (km/h). Dia 18/10/2017 de OOA 24 UTC

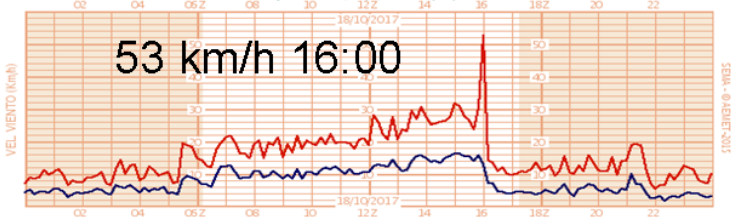

Valls: $118 \mathrm{~km} / \mathrm{h}$ a $16: 07$ UTC

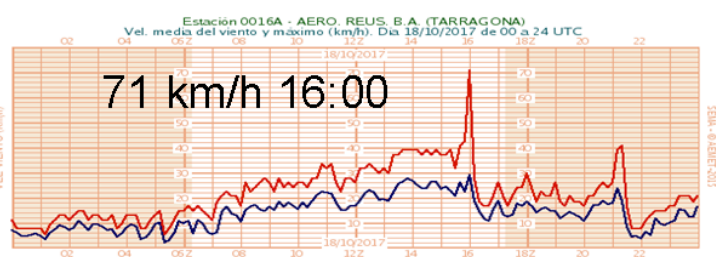

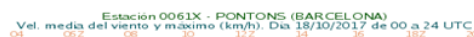

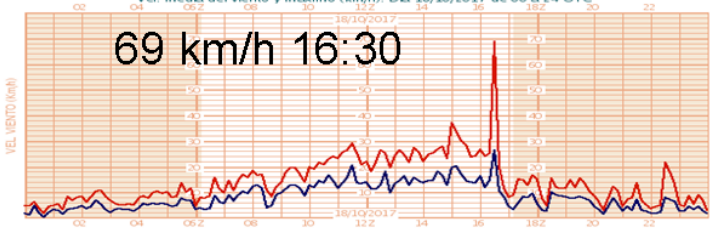

Figura 1. Evolución temporal de la temperatura del aire, el punto de rocío, la velocidad media del viento y el valor de la racha máxima en 4 EMA de la zona de interés. Se indica también la hora (UTC) y la velocidad $(\mathrm{km} / \mathrm{h})$ de la racha máxima en esos puntos. (Para Valls no se dispone de datos de viento de la propia estación de AEMET sino de una estación cercana).

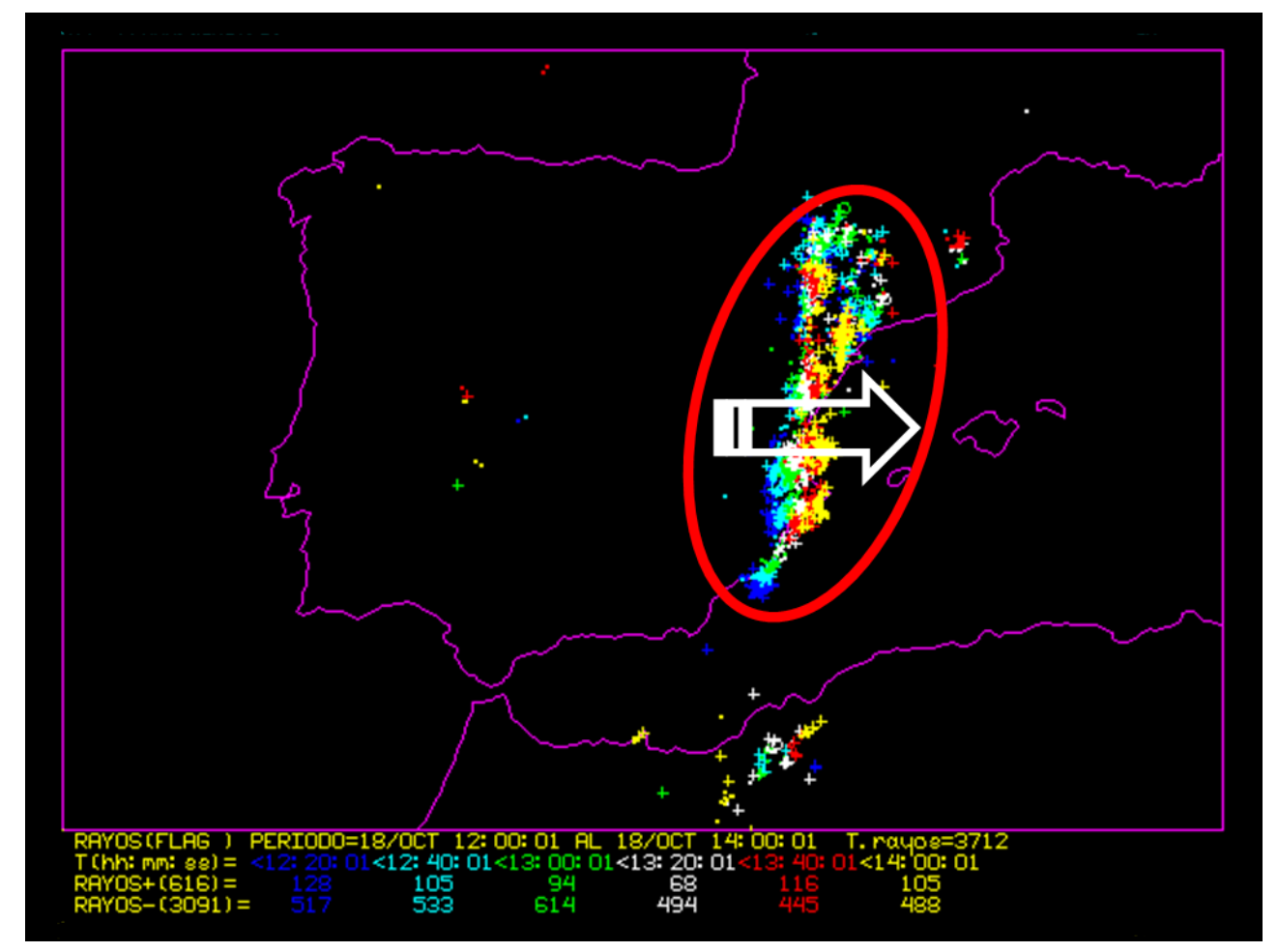

Figura 2. Rayos NT registrados entre las 12 y las 14 UTC en el entorno de la península ibérica. 


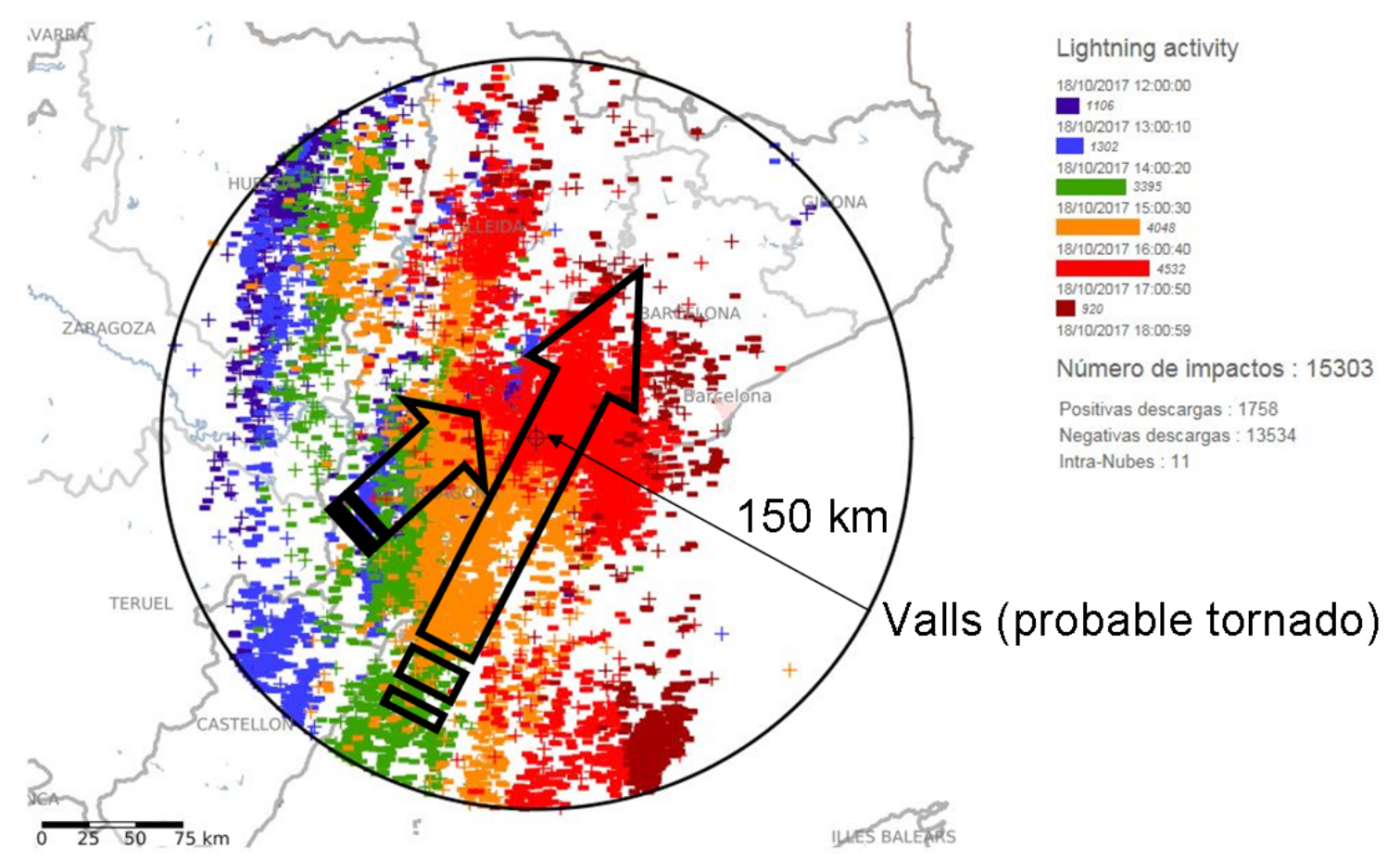

Figura 3. Impactos de descargas eléctricas registrados entre las 12 y las 18 UTC en el entorno de la zona de estudio (se debe tener en cuenta que cada rayo puede producir varias descargas eléctricas).

En un círculo de $150 \mathrm{~km}$ de radio, centrado en la localidad de Valls, que engloba la zona de estudio, se registró un altísimo número de impactos de descargas eléctricas: 15303 entre las 12 y las 18 UTC, la gran mayoría de estas ligadas a la estructura convectiva en línea que produjo los chubascos y los vientos fuertes. La distribución espaciotemporal de estos impactos (fig. 3) muestra claramente el avance del MCS de sudoeste a nordeste, dirección de avance distinta a la del frente frío (oeste-este).

La serie quinceminutal de impactos en un círculo de radio $30 \mathrm{~km}$ en torno a Valls, que engloba la mayoría de los lugares afectados por los vientos fuertes, muestra unos valores muy altos especialmente entre las 15:45 y las 16:30 UTC (tabla 1). Entre las 15:15 y las 16:00 UTC se produjo un incremento muy grande en el número de impactos que respondía a la dinámica interna de la estructura convectiva. Estos cambios son citados en la bibliografía con el nombre de lightning jump (p. ej. FARNELL et al., 2016) y parecen estar vinculados con la inminente aparición de fenómenos severos en superficie (granizo grande, rachas fuertes de viento, tornados).

\begin{tabular}{|c|c|}
\hline $\begin{array}{c}\text { Intervalo } \\
\text { horario } \\
\text { (UTC) }\end{array}$ & $\begin{array}{c}\text { Número de impactos } \\
\text { y porcentaje de incremento } \\
\text { respecto al periodo anterior }\end{array}$ \\
\hline $15: 15-15: 30$ & 7 \\
\hline $15: 30-15: 45$ & $117(1571 \%)$ \\
\hline $15: 45-16: 00$ & $1058(804 \%)$ \\
\hline $16: 00-16: 15$ & $1086(3 \%)$ \\
\hline $16: 15-16: 30$ & $722(-34 \%)$ \\
\hline $16: 30-16: 45$ & $19(-97 \%)$ \\
\hline
\end{tabular}

Tabla 1. Descargas eléctricas registradas en torno a la localidad de Valls en periodos de 15 minutos. 


\section{IMPACTOS}

El evento del 18 de octubre tuvo un impacto relevante en Cataluña, especialmente en muchas comarcas de la provincia de Tarragona, donde se produjo la línea de turbonada. En dicha provincia hubo 14 municipios afectados (3 solo por lluvia en Terra Alta, y 11 por viento y lluvia), siendo las afectaciones más importantes en las comarcas de Camp de Tarragona y en Terres de l'Ebre, donde se produjeron graves desperfectos y heridos. El servicio de Emergencias de la Generalitat de Cataluña (112) recibió más de 500 llamadas, y el servicio de bomberos, 104 avisos. Hubo decenas de árboles caídos y ramas rotas, que a su vez provocaron varios heridos. Un árbol cayó sobre la AP-7, y se produjeron cortes en varias carreteras secundarias, así como en el servicio de trenes (el Euromed a la altura de Tarragona, y la línea R-15 de Ferrocarrils de Catalunya). El mobiliario urbano de varios municipios fue movido, levantado, tumbado y arrancado, y hubo importantes daños

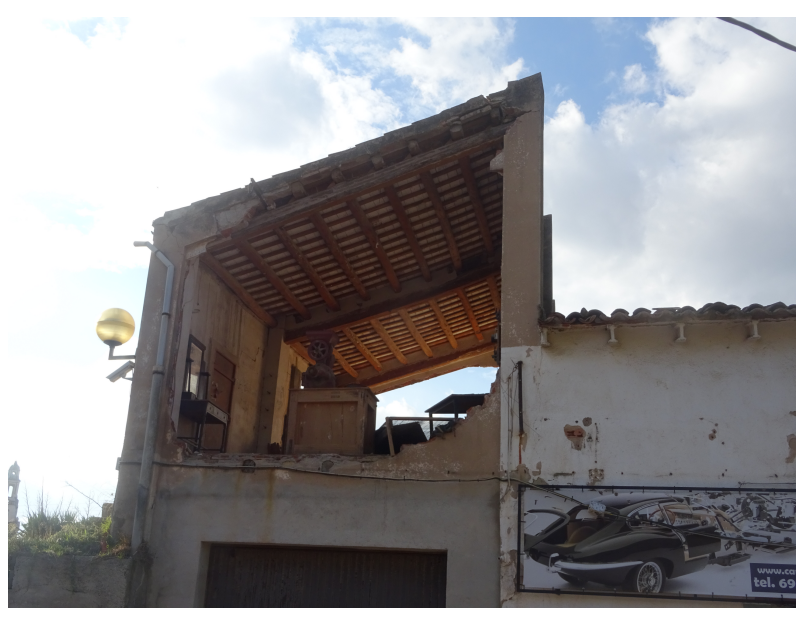

Figura 4. Fotografía del segundo piso de un taller de coches en Valls cuyas paredes laterales fueron arrancadas durante el tornado. Una de las paredes cayó sobre un vehículo estacionado en la calle (autor: Oriol Rodriguez, Universitat de Barcelona). materiales, con afectaciones al puerto y al circo y pretorio romano de la ciudad de Tarragona. Una residencia de ancianos en Gandesa se inundó, aunque afortunadamente no hubo que lamentar víctimas. El suministro eléctrico sufrió cortes en al menos 5 municipios, afectando a más de 1100 abonados.

La población de Valls (Alt Camp) fue una de las más afectadas por las fuertes tormentas de la jornada. A las 16 UTC se produjo la tormenta más intensa, asociada a un frente de racha o reventón (con un probable tornado, BECH et al., 2018), que produjo múltiples daños. El intenso viento arrancó la fachada de un edificio (http://www.elvallenc.cat/video/el-pas-del-tornado-per-valls/). Hubo 13 heridos leves, al menos 150 árboles caídos, una cubierta de un polideportivo arrancada, cortes en el suministro eléctrico de algunos barrios, y afectaciones en comercios, estación de autobuses, farolas, parkings y señales de tráfico (fig. 4).

\section{SITUACIÓN A ESCALA SINÓPTICA Y MESOESCALAR}

El entorno sinóptico del día 18 de octubre estuvo dominado en niveles medios y altos por una profunda vaguada atlántica (en 500 y $300 \mathrm{hPa}$ ) extendida desde el oeste de Irlanda hasta el sur de Marruecos, con eje NW-SE a las 12 UTC y un mínimo casi aislado de temperatura en $500 \mathrm{hPa}$ de $-21^{\circ} \mathrm{C}$ (protodana). Dicha vaguada (fig. 5) avanzaba lentamente hacia el Mediterráneo, mientras que la mitad este de la Península y

a)

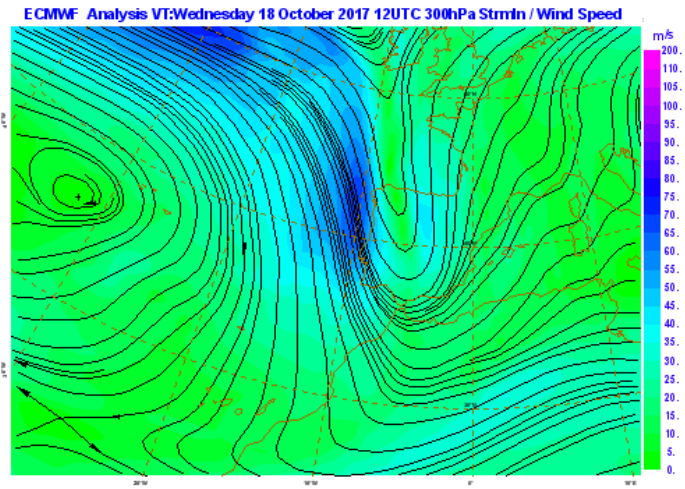

b)

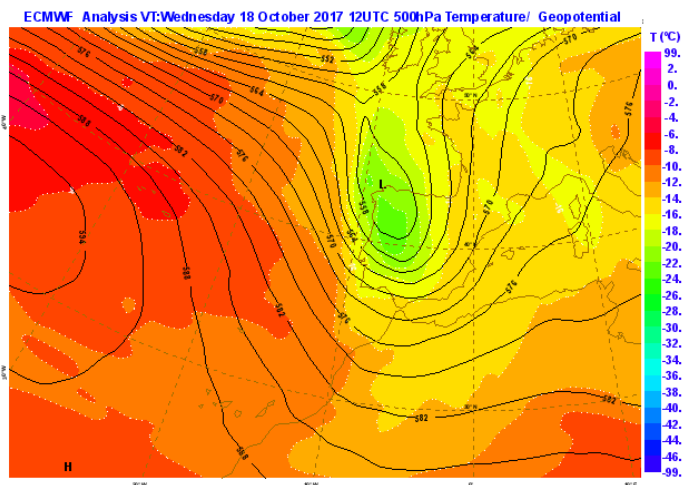

Figura 5. Análisis del 18 de octubre a 12 UTC: a) líneas de corriente y velocidad del viento en $300 \mathrm{hPa}$; b) $\mathrm{T}$ y altura geopotencial en $500 \mathrm{hPa}$. 
Baleares quedaban bajo la rama ascendente de dicha vaguada. De este modo, sobre el nordeste peninsular el flujo del sur moderado difluente (difluencia a la salida de la vaguada) aportó forzamiento dinámico para el desarrollo de la convección. En $300 \mathrm{hPa}$ (fig. 5a), la vaguada también estaba presente con un desequilibrio entre su chorro trasero (jet streak de $100 \mathrm{kt}$ aumentando a 120 a las 12 UTC) y su chorro delantero (también aumentado de 50 a 75 kt), formándose la estructura convectiva a la derecha de este último (fig. 6a). El desequilibrio entre chorros fue probablemente responsable del lento avance de la vaguada.

En superficie, el anticiclón atlántico se localizaba estacionario al suroeste de las islas Azores sin afectar a la península ibérica, mientras que dos bajas atlánticas se situaban sobre el oeste de Irlanda y otra sobre Bretaña a las 00 UTC, desplazándose durante el día hacia el canal de la Mancha. Llevaban asociados dos frentes fríos que se situaban a las 12 UTC en la mitad oriental peninsular (fig. 6b), con desplazamiento hacia el este. Estos frentes sirvieron de mecanismo de disparo de la convección primaria. Sobre el levante peninsular se situaba una masa húmeda (alta humedad relativa en 850 y $700 \mathrm{hPa}$ ), debido en parte al flujo moderado a fuerte de componente sur sobre Cataluña, que también provocó el rápido desplazamiento de las estructuras convectivas.

a)

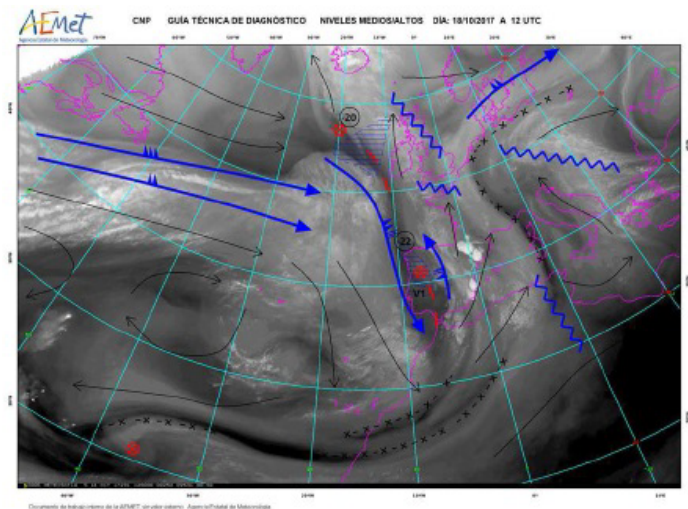

b)

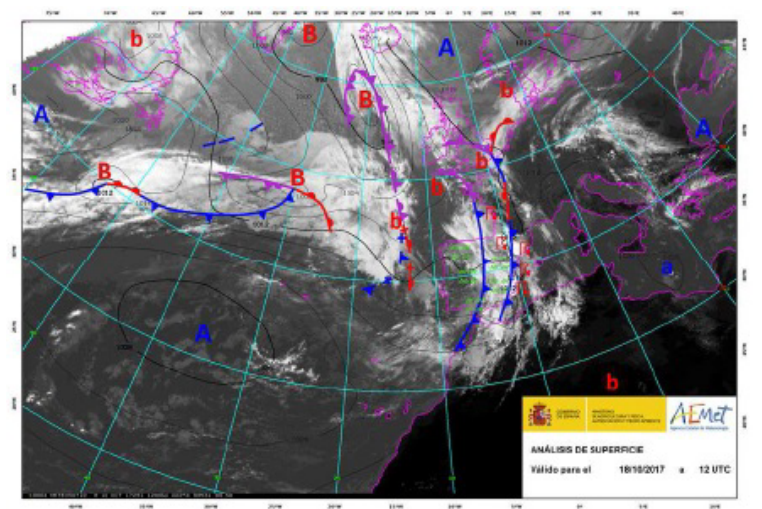

Figura 6. a) Imagen canal WV6.2 Meteosat10 con diagnóstico en niveles medios/altos superpuesto (12 UTC); b) Imagen IR 10.8 Meteosat10 + análisis frontal (12 UTC).

En 850 hPa también estaba presente una amplia y profunda vaguada que afectaba a toda la península, avanzando hacia el este (dando lugar a un flujo medio del suroeste flojo), y una vaguada térmica ligeramente retrasada respecto a la vaguada de altura geopotencial (no mostrado). En el nordeste la temperatura a ese nivel era de $11-12^{\circ} \mathrm{C}$ a las $12 \mathrm{UTC}$, propiciando un importante contraste con la temperatura a $500 \mathrm{hPa}$ (unos 26-27 ${ }^{\circ} \mathrm{C}$ de diferencia). Dicha masa relativamente cálida en $850 \mathrm{hPa}$ se mantenía a las 18 UTC sobre el nordeste mientras que la incursión fría de la vaguada ya era notable, habiendo avanzado la masa fría hacia el Mediterráneo y alcanzado Valencia a esa hora $\left(9^{\circ} \mathrm{C}\right)$.

A nivel mesoescalar, el flujo medio en $925 \mathrm{hPa}$ era del sureste desde el norte de Castellón hasta el sur de Barcelona (fig. 7), con un efecto de canalización del viento por el valle del Ebro. Los sondeos observados (Barcelona, Palma) y previstos por HRES-IFS ECMWF (puntos sobre el aeropuerto de Lleida-Alguaire, aeropuerto de Reus, y aeropuerto de Castellón) reflejaban una inestabilización general en el área entre las 00 y las 12 UTC, produciéndose un aumento considerable de índices de inestabilidad como el SBCAPE y el índice K (tabla 2 y fig. 8b). En la mesoescala $\alpha$ se observó un lóbulo de inestabilidad en el valle del Ebro (fig. 8a).

Figura 7.

Análisis del campo de viento y humedad relativa

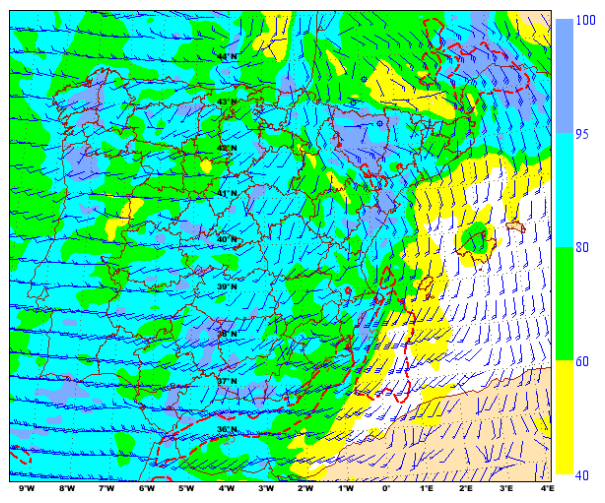

(HRES-ECMWF, 12 UTC). 


\begin{tabular}{|c|c|c|c|c|}
\hline Punto & K & $\pi$ & LI & SBCAPE \\
\hline $\mathrm{BCN}$ & $28,1 \gg>>32,6$ & $43,3>>>52,3$ & $-1,1>>>-3,9$ & 249,3 >>> 1812,1 \\
\hline PMA & $21,2 \gg \gg>25,4$ & $43,7>>>43,0$ & $-1,5>>>-3,4$ & $0,0 \gg>1820,0$ \\
\hline LEDA & $32,7 \gg>>34,2$ & $48,7>>>51,8$ & $-0,6>>>-2,9$ & $67,7>>>988,4$ \\
\hline LERS & $32,9>>>32,6$ & $50,7 \gg>>50,5$ & $-2>>>-3,1$ & 561,3 >>> 1017,6 \\
\hline LECH & $32,2>>>34,5$ & $50,8 \gg>>50,6$ & $-2>>>-1,6$ & $38,0 \gg>>666,8$ \\
\hline $\begin{array}{l}\text { K = (T8 } \\
\text { POSIBIL } \\
<15 \text { Al } \\
{[15-20} \\
{[21-25} \\
{[26-30} \\
{[31-35} \\
{[36-4 C} \\
>40 \mathrm{Ce}\end{array}$ & $\begin{array}{l}50 \text { ROCÍO) - (T7 } \\
\text { ENTAS DE MASA }\end{array}$ & o Rocío) & & \\
\hline $\begin{array}{l}\text { TT }=(T \mathcal{E} \\
\text { INTENS } \\
\text { Tormer } \\
\text { Tormen } \\
\text { Tormen } \\
\text { Tormen } \\
\text { Tormen } \\
\text { Tormen }\end{array}$ & $\begin{array}{l}\text { ÍO) - } 2 \text { (T500) } \\
\text { URA ESPACIAL DE } \\
8 \text { a } 52 \\
\text { algunas de inter } \\
\text { algunas modera } \\
\text { dispersas, algu } \\
\text { moderadas, alg } \\
\text { moderadas, cor }\end{array}$ & $\begin{array}{l}\text { RMENTAS. VALO } \\
\text { noderada: } 52 \text { a } \\
\text { lguna aislada s } \\
\text { eras: } 58 \text { a } 61 \\
\text { era: } 61 \text { a } 64 \\
\text { ntas severas: > }\end{array}$ & $\begin{array}{l}\text { DTAL DE TOTALES } \\
5 \text { a } 58\end{array}$ & \\
\hline \multicolumn{5}{|c|}{ 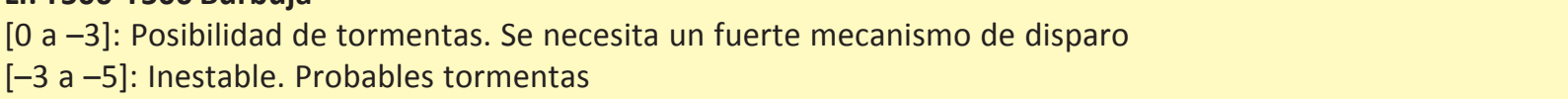 } \\
\hline
\end{tabular}

Tabla 2: Evolución entre las 00 y las 12 UTC de los índices de inestabilidad K, Total de Totales (TT), Lifted Index (LI) y Surface Based Convective Available Potential Energy (SBCAPE) obtenidos de sondeos observados en Barcelona (BCN) y Palma de Mallorca (PMA) y previstos por el HRES-IFS ECMWF para puntos sobre los aeropuertos de Lleida-Alguaire (LEDA), Reus (LERS) y Castellón (LECH). Abajo, leyenda sobre los distintos índices y su significado convectivo.

La combinación del forzamiento sinóptico-frontal (subsinóptico) y algún tipo de forzamiento por convergencia local dio lugar al desarrollo de convección profunda y organizada, con la formación de la línea de turbonada que se analiza en el siguiente punto.

a)

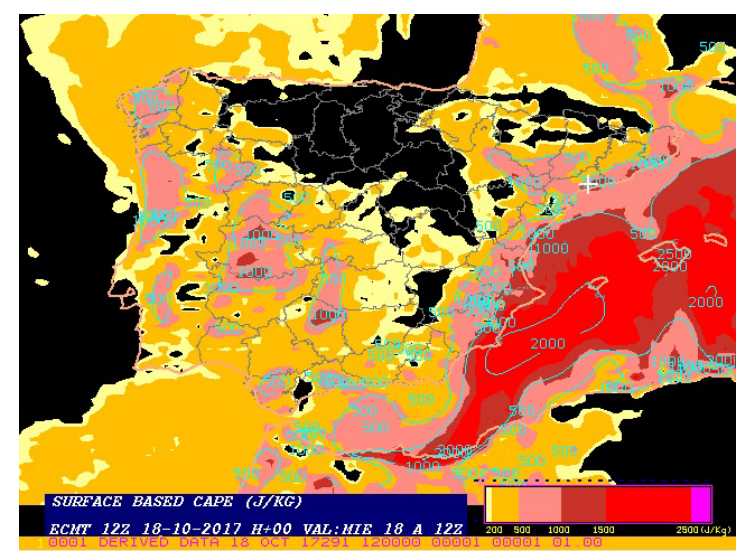

b)

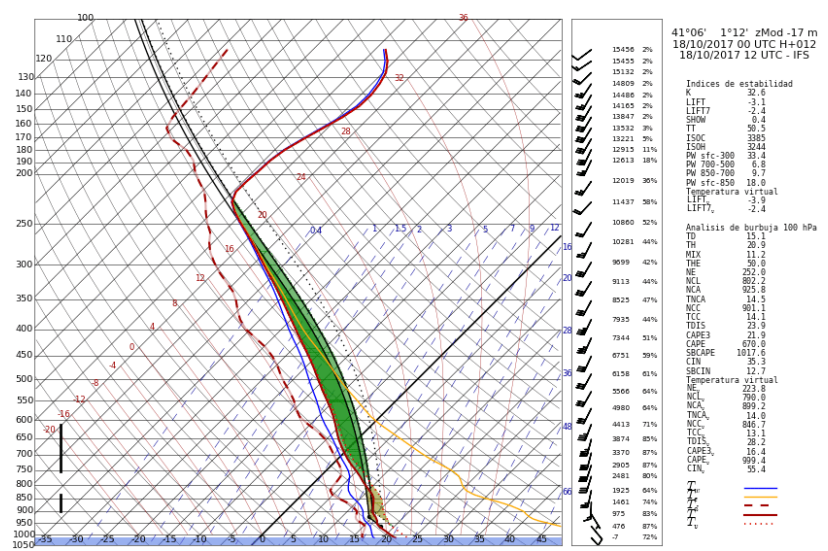

Figura 8. a) SBCAPE previsto (HRES-ECMWF, 00 UTC) a las 12 UTC;

b) sondeo previsto (HRES-ECMWF, 00 UTC) para el aeropuerto de Reus a las 12 UTC. 


\section{ESTRUCTURAS CONVECTIVAS}

El campo de nubosidad en el que se desarrolló el sistema convectivo de interés era muy complejo, de manera que la intensa convección frontal resultaba embebida. Aunque la convección más intensa se desarrolló por la tarde, parece que el papel que jugó la radiación solar en el disparo de la convección fue secundario, primando el forzamiento frontal como generador de la convección primaria.

El nacimiento y posterior organización de la línea convectiva principal, en forma de línea de turbonada (squall line) (SCHOEN y Ashley, 2011), se produjo a partir de un grupo de células convectivas independientes pero cercanas localizadas en el sur y el sudoeste de la provincia de Tarragona. Este tipo de inicio recibe el nombre de broken areal (ÁlvAREZ et al., 2018). A las 16:00 UTC (fig. 9) la imagen realzada del canal IR 10,8 $\mu \mathrm{m}$ del Meteosat10 muestra un MCS principal cubriendo el sur y el oeste de Cataluña y el este de Aragón y un segundo sistema de menor tamaño al sur del primero, sobre el mar. El realce usado (PREVIMET) muestra que las temperaturas de brillo ( $\mathrm{Tb}$ ) en los topes nubosos del sistema convectivo principal alcanzaban valores muy bajos, del orden de -64 a $-68^{\circ} \mathrm{C}$, indicadores de la gran profundidad de la convección.

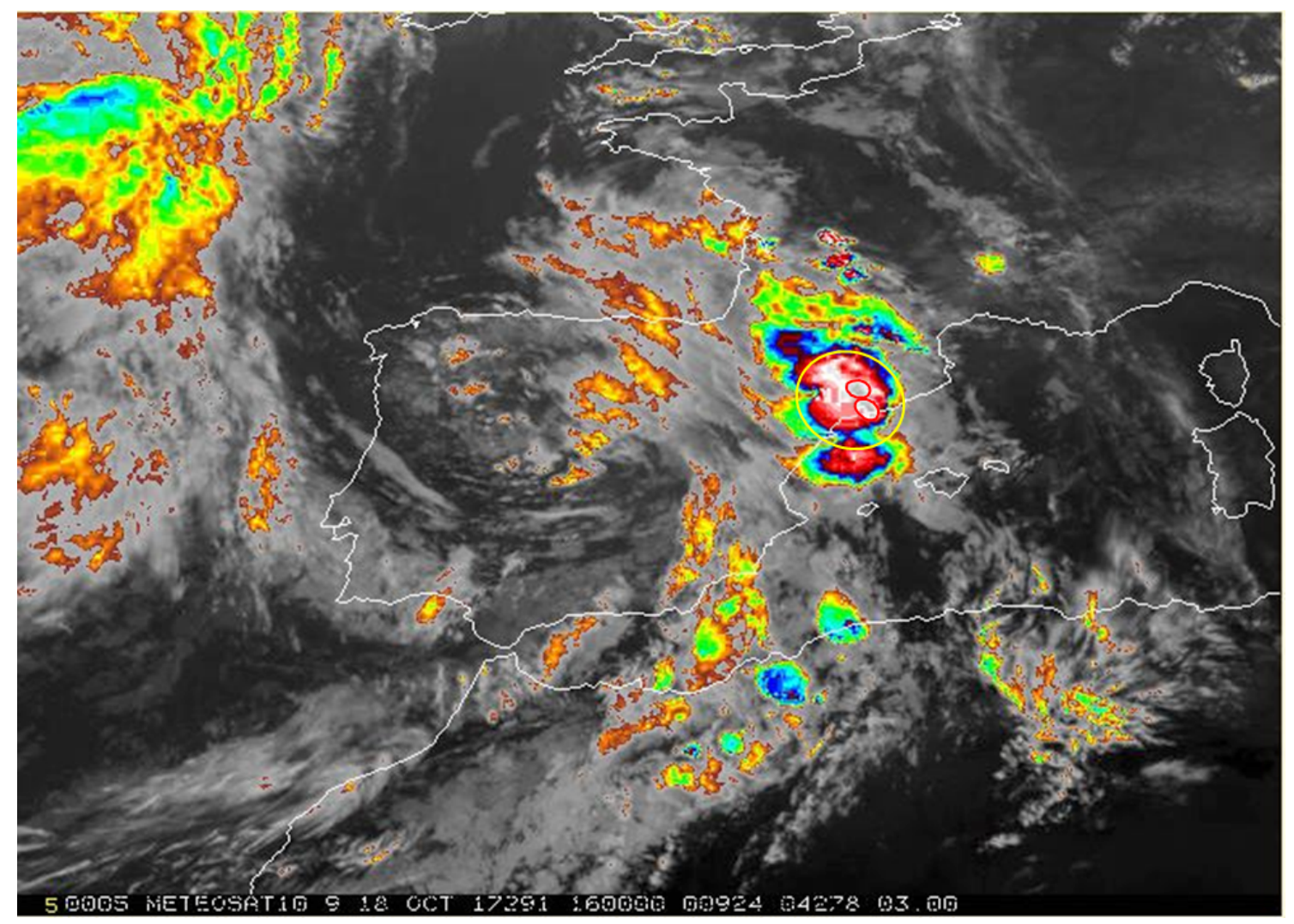

Figura 9. Imagen realzada del canal 10,8 $\mu \mathrm{m}$ del Meteosat10 de las 16 UTC.

El círculo amarillo engloba el MCS de interés. Realce PREVIMET $\left(\mathrm{Tb}\right.$ en $\left.{ }^{\circ} \mathrm{C}\right)$ : marrón: -32/-36; naranja: -36/-40; amarillo: -40/-44; verde: -44/-48; azul cielo: -48/-52; azul marino: -52/-56; rojo: -56/-60; blanco: -60/-64; gris: -64/-68; negro: -68/-80.

Aunque la estructura convectiva principal interaccionó con otros sistemas convectivos desarrollados más al oeste y ello dificulta la delimitación precisa de su forma, algunas de las imágenes radar disponibles, por ejemplo, el PPI0 (haz radar a 0,5 de inclinación) de las 15:30 UTC (fig. 10), parecen mostrar que la línea de turbonada tenía un área estratiforme posterior (stratiform squall line), que es una de las características más habituales en este tipo de MCS. 


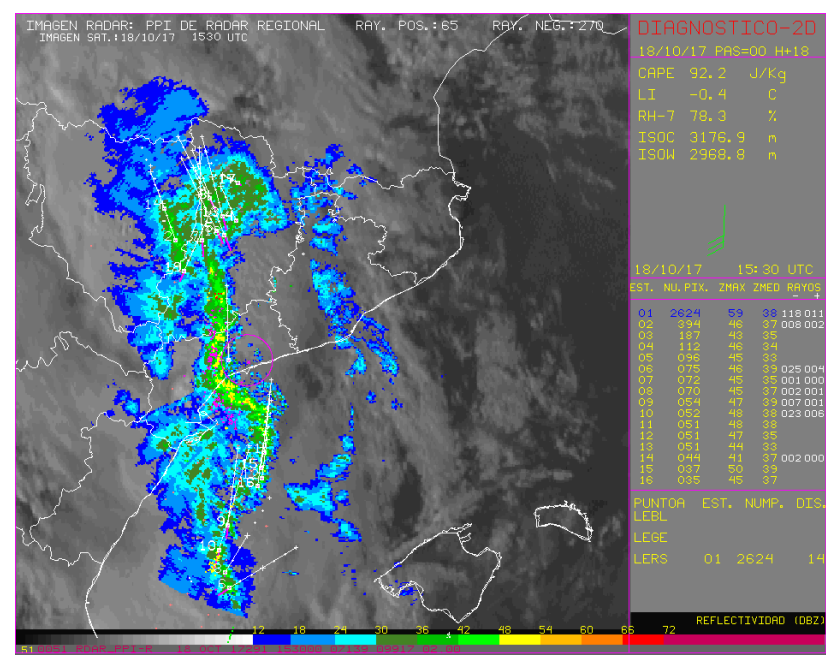

Figura 10. Imagen de las 15:30 UTC del PPI0 del radar de AEMET en Cataluña. Se incluye también en la imagen el diagnóstico radar 2D (YRADAR2D; SAN Ambrosio, 2011).

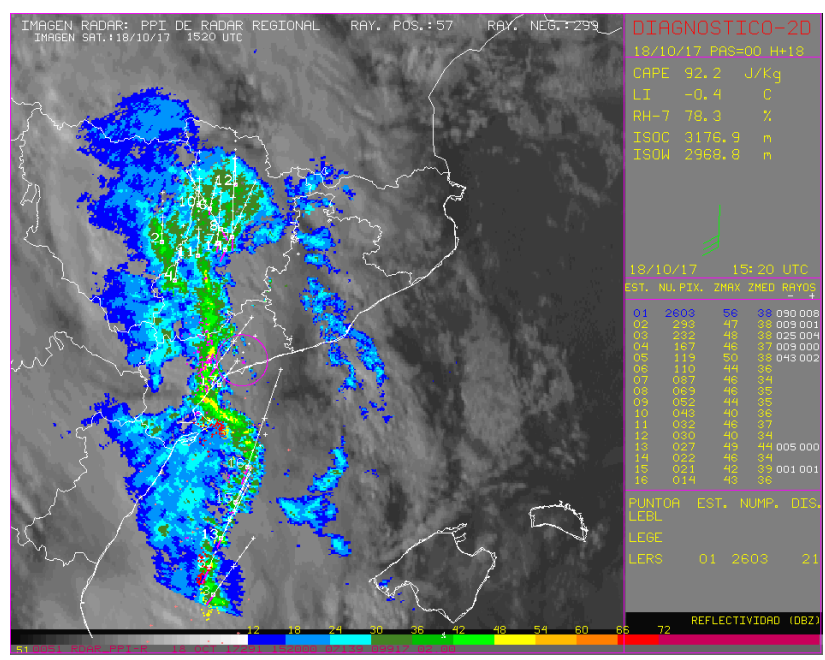

Figura 11. Imagen de las 15:30 UTC del PPI0 del radar de AEMET en Cataluña. Se incluye también en la imagen el diagnóstico radar 2D.

El análisis del bucle de imágenes del PPI0 del radar de AEMET en Cataluña permite establecer que la línea de turbonada fue trasladándose primero hacia el oeste y posteriormente hacia el nordeste durante unas 3-4 horas, desde las 13:00 hasta las 16:50 UTC, cuando alcanzó el sur de la provincia de Barcelona y perdió entidad. La traslación fue relativamente lenta y perpendicular a su eje mayor. Este eje fue basculando en sentido antihorario de manera que su extremo sur avanzó una distancia bastante mayor que el norte. Este hecho se explica por la aparición hacia las 15:20 UTC de un eco intenso en forma de arco (bow echo) (W Акімото, 2001) (fig. 11) en el extremo sur de la línea de turbonada, en la zona del delta del Ebro, que avanzó rápidamente impelido por un paquete de viento huracanado identificado por primera vez en ese área en el campo de viento radial Doppler a las 15:10 UTC (fig. 12). Dado que esa zona se encuentra a unos $120 \mathrm{~km}$ del radar se estima que esa señal se podría encontrar a unos 1-2 km de altitud.

La velocidad radial estimada del viento en ese paquete era de unos $173 \mathrm{~km} / \mathrm{h}$, lo cual provocó el salto de escala (ambigüedad) en la representación gráfica de esos valores. Ese paquete de viento se mantuvo bien identificable durante unos 60 minutos, trasladándose rápidamente hacia el norte y siendo probablemente el responsable de las fuertes rachas de viento registradas en el centro de la provincia de Tarragona. El origen de este máximo de viento en el límite entre la mesoescala $\alpha$ y la $\beta$, generador del bow echo, podría ser un reventón (macroburst) asociado a las células convectivas especialmente intensas ( $54 \mathrm{dBZ}$ ) que había en el extremo sur de la línea de turbonada.

La línea de turbonada quedó bien establecida hacia las 13:00 UTC, tuvo un ciclo de vida de unas 3-4 horas de duración y se trasladó unos $150 \mathrm{~km}$. En su momento de máxima extensión medía unos $200 \mathrm{~km}$ de longitud (límite entre la mesoescala $\alpha$ y la $\beta$ ), la temperatura de brillo de los topes nubosos alcanzó los $-68^{\circ} \mathrm{C}$, el echotop máximo (para umbral $12 \mathrm{dBZ}$ ) llegó hasta los $15,5 \mathrm{~km}$, la reflectividad máxima en el PPI0 fue de $59 \mathrm{dBZ}$ y la DVIL máxima de $3 \mathrm{~g} / \mathrm{m}^{3}$. Todos estos valores son compatibles con la severidad de los fenómenos observados en superficie y con la elevadísima frecuencia de rayos NT.

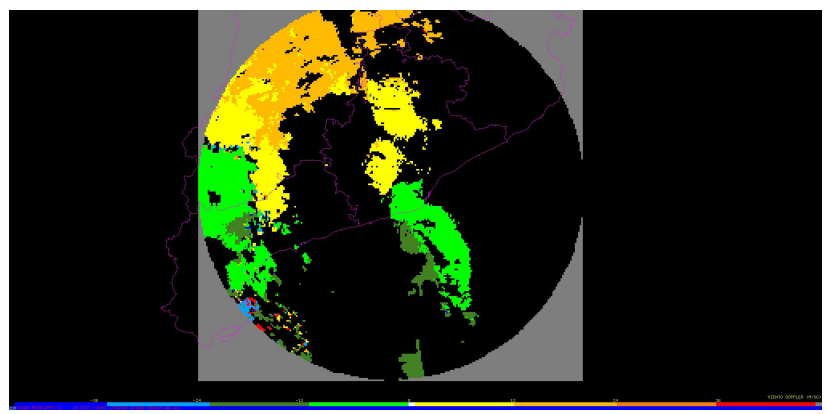

Figura 12. Imagen de velocidad radial Doppler del radar de AEMET en Cataluña de las 15:10 UTC. Colores cálidos indican alejamiento del radar y fríos acercamiento al radar. 


\section{AVISOS DE FENÓMENOS METEOROLÓGICOS ADVERSOS EMITIDOS}

El Plan Nacional de Predicción y Vigilancia de Fenómenos Meteorológicos Adversos (Meteoalerta) de AEMET pretende facilitar la más detallada y actualizada información posible sobre los fenómenos atmosféricos adversos que puedan afectar a España hasta un plazo máximo de 60 horas, así como proporcionar una información continuada de su evolución una vez que han iniciado su desarrollo (http://www.aemet.es/documentos/es/ eltiempo/prediccion/avisos/plan_meteoalerta/plan_meteoalerta.pdf). Dentro del plan Meteoalerta, los umbrales de aviso de fenómenos meteorológicos adversos por precipitación, para las distintas zonas afectadas en la provincia de Tarragona pueden verse en la siguiente tabla:

\begin{tabular}{|l|c|c|c|c|cc|}
\hline \multirow{2}{*}{ NOMBRE DE LA ZONA } & \multicolumn{6}{|c|}{ Umbrales } \\
\cline { 2 - 7 } & \multicolumn{3}{|c|}{ Precipitación 12 h (P2) } & \multicolumn{3}{c|}{ Precipitación 1 h (P1) } \\
\cline { 2 - 7 } & amarillo & naranja & rojo & amarillo & naranja & rojo \\
\hline Prelitoral norte de Tarragona & 60 & 100 & 180 & 20 & 40 & 90 \\
\hline Prelitoral sur de Tarragona & 60 & 100 & 180 & 20 & 40 & 90 \\
\hline Litoral norte de Tarragona & 60 & 100 & 180 & 20 & 40 & 90 \\
\hline Litoral sur de Tarragona & 60 & 100 & 180 & 20 & 40 & 90 \\
\hline
\end{tabular}

Tabla 3. Umbrales de avisos de precipitación por zonas de aviso en Tarragona.

Los avisos por tormenta se emiten cuando se espera que, además de las descargas eléctricas, se produzcan lluvias y vientos fuertes (incluyendo tornados) y granizo.

El día previo al episodio, el martes 17 a las 11:17 H. O., AEMET emitió avisos de nivel naranja de tormenta y precipitación acumulada en 1 hora (TO y P1) para las zonas de Litoral Norte y Prelitoral Norte de Tarragona, y de nivel amarillo (TO y P1) para el resto de la provincia de Tarragona (Litoral Sur y Prelitoral Sur) desde las 8 hasta las 24 H. O. del día 18, con comentarios acerca de la incertidumbre en la zona y la intensidad. El miércoles 18 a las 13:31 H. O., a raíz de las afectaciones del episodio, se decidió subir a nivel naranja de aviso de TO y P1 las zonas de Litoral y Prelitoral Sur, quedando toda la provincia en nivel naranja hasta las 24 H. O. de ese mismo día.

\section{CONCLUSIONES}

El análisis realizado del episodio de tiempo muy adverso en Cataluña del 18 de octubre de 2017 muestra una jerarquía espaciotemporal de estructuras meteorológicas generadoras de los diferentes fenómenos: vaguada, frentes fríos, línea de turbonada (MCS) y bow echo, cada una de ellas englobando a la de menor tamaño. Tanto las salidas de los modelos numéricos de predicción como las herramientas de teledetección se muestran cruciales para la comprensión completa del episodio.

Este episodio ejemplifica el hecho de que no son incompatibles bajo un mismo entorno los clásicamente llamados fenómenos severos, en este caso vientos muy fuertes y probable tornado, con las lluvias torrenciales, asociadas a procesos de precipitación altamente eficientes.

Los fenómenos meteorológicos observados han tenido numerosos impactos, repartidos en un área relativamente amplia de la provincia de Tarragona debido al tamaño mesoescalar de la estructura convectiva principal, aunque las estructuras de escala menor (bow echo) han concentrado los mayores efectos en un área lógicamente más reducida. 
Aunque la situación meteorológica general fue muy bien prevista desde el día anterior, la constancia de efectos significativos en superficie en alguna de las zonas de aviso implicó la elevación del nivel de aviso en esas zonas. Probablemente, la identificación precoz del bow echo y del paquete de viento muy fuerte en las imágenes de viento Doppler podría haber servido para adelantar alguna hora la emisión del aviso naranja en las zonas posteriormente afectadas por los vientos más fuertes. Este hecho pone de manifiesto la importancia de las tareas de nowcasting en los episodios altamente convectivos.

\section{AGRADECIMIENTOS}

Se quiere expresar un especial agradecimiento a todas las unidades de AEMET que hacen posible este tipo de estudios.

\section{REFERENCIAS}

Álvarez, E., Espejo, F., Cortés, F. J., Lafragüeta, C. y Serrano, R., 2011. Caracterización sinóptica de los procesos convectivos en el interior del nordeste peninsular. Nota Técnica . $^{\mathrm{o}} 3$ de la Delegación Territorial en Aragón. Agencia Estatal de Meteorología. NIPO: 784-11-008-8.

Álvarez, R., Roa, A., García, M. T., López, A., Cano, D. y Ayensa, E., 2018. Línea de turbonada en la península ibérica. XXXV Jornadas Científicas de la Asociación Meteorológica Española. León, 5-7 de marzo de 2018.

Bech, J., Rodríguez, O., Altube, P., Rigo, T., Pineda, N., Castán, S., Arús, J. y Montanyá, J., 2018. Doppler radar observations of two tornadic thunderstorm cases in the western mediterranean region. ERAD 2018. Utrecht (Holanda), 1-6 de julio de 2018.

Davies-Jones, R., 2015. A review of supercell and tornado dynamics. Atmos. Res., 158-159, 274-291.

Doswell III, C. A., Ramis, C., Romero, R. y Alonso, S., 1998: A Diagnostic Study of Three Heavy Precipitation Episodes in the Western Mediterranean Region. Wea. Forecasting, 13, 102-124.

Farnell, C., Rigo, T. y Pineda, N., 2016. Lightning jump as a nowcast predictor: Application to severe weather events in Catalonia. Atmospheric Research, 1, pp. 38-46.

Houze, R. A., 2004. Mesoscale convective systems. Rev. Geophys., 42, RG4003, doi: 10.1029/ 2004RG000150.

Kuchera, E. L. y PARKer, M.D., 2006. Severe convective wind environments. Weather and Forecasting, 21, pp. 595-512.

Llasat, M. C., Llasat-Botija, M., Rodríguez, A. y Lindbergh, S., 2010. Flash floods in Catalonia: a recurrent situation. Adv. Geosci., 26, 105-111.

Mateo, J., Ballart, D., Brucet, C., Aran, M. y Bech, J., 2009. A study of a heavy rainfall event and a tornado outbreak during the passag of a squall line over Catalonia. Atmos. Research, 93, 131-146.

Pascual, R., Cuevas, G. y González, S., 2015. Estudio de la situación de tiempo adverso del 1 y 2 de agosto de 2014 en el nordeste peninsular. Nota técnica n. ${ }^{\circ} 16$ de la Delegación Territorial de AEMET en Cataluña. NIPO: 281-15-006-3.

Riesco, J., Mora, M., de Pablo F. y Rivas, L., 2014. Regimes of intense precipitation in the Spanish Mediterranean area. Atmos. Research, 137, 66-79. 
Riosalido, R., Eliazaga, F., CARretero, O. y F. Martín, 1998. Climatología satélite de sistemas convectivos de mesoescala en las proximidades de la península ibérica: Aplicación a la predicción de lluvias torrenciales. Nota Técnica STAP n. ${ }^{\circ}$ 29. INM. Documento interno.

Romero, R., Ramis, C., Alonso, S., Doswell III, C.A. y Stensrud, D. J., 1998. Mesoscale Model Simulations of Three Heavy Precipitation Events in the Western Mediterranean Region. Mon. Wea. Rev., 126, 1859-1881.

Romero, R., Sumner, G., Ramis, C. y Genovés, A., 1999. A Classification of the atmospheric circulation patterns producing significant daily rainfall in the Spanish Mediterranean area. Int. J. Climatol., 19, 765-785.

SAN Ambrosio, I., 2011. Aplicación para la caracterización objetiva de estructuras y células convectivas mediante radar regional. Documento interno AEMET.

Schoen, J. M. y Ashley, W. S., 2011. A climatology of fatal convective winds events by storm type. Weather and Forecasting, 26, pp. 109-121.

Waкimoto, R., 2001. Convectively driven high wind events. En: Doswell III., C.A. (Ed.). Severe Convective Storms. American Meteorological Society, pp. 255-298. 
